\title{
Inexact Newton-Type Methods for Non-Linear Problems Arising from the SUPG/PSPG Solution of Steady Incompressible Navier-Stokes Equations
}

A. L. G. A. Coutinho and

M. A. D. Martins

Center for Parallel Computations and Department

of Civil Engineering

Federal University of Rio de Janeiro

P. O. Box 68506

21945-970 Rio de Janeiro, R

Rio de Janeiro, RJ. Brazil

\{renato, alvaro, marcos\}@nacad.ufj.br

The finite element discretization of the incompressible steady-state Navier-Stokes equations yields a non-linear problem, due to the convective terms in the momentum equations. Several methods may be used to solve this non-linear problem. In this work we study Inexact Newton-type methods, associated with the SUPG/PSPG stabilized finite element formulation. The resulting systems of equations are solved iteratively by $a$ preconditioned Krylov-space method such as GMRES. Numerical experiments are shown to validate our approach. Performance of the nonlinear strategies is accessed by numerical tests. We concluded that Inexact Newton-type methods are more efficient than conventional Newton-type methods.

Keywords: Inexact Newton-type methods, Newton-Krylov methods, Navier-Stokes, incompressible fluid flow, finite elements

\section{Introduction}

We consider the simulation of incompressible fluid flow governed by Navier-Stokes equations using the stabilized finite element formulation proposed by Tezduyar (1991). This formulation allows that equal-order-interpolation velocity-pressure elements are employed, circumventing the Babuska-Brezzi stability condition by introducing two stabilization terms. The first term is the Streamline Upwind Petrov-Galerkin (SUPG) presented by Brooks \& Hughes (1982) and the other one is the Pressure Stabilizing Petrov Galerkin (PSPG) stabilization proposed initially by Hughes et al (1986) for Stokes flows and later generalized by Tezduyar (1991) to finite Reynolds number flows. SUPG/PSPG implementations have been very successful in the simulation of complex, large scale fluid flow problems in parallel computers (Tezduyar et al, 1993, 1996). Nowadays it has been used for simulations of 3D fluid-structure interaction problems on unstructured grids with more than one billion of elements (Aliabadi et al, 2002). Usually the resulting fully coupled (velocity-pressure) linearized systems of equations are solved by a preconditioned Krylov-space iterative method such as GMRES (Saad, 2003).

If we restrict ourselves to the steady case, it is known that, when discretized, the incompressible Navier-Stokes equations give rise to a system of nonlinear algebraic equations due the presence of convective terms in the momentum equations. Among several strategies to solve nonlinear problems the Newton's methods are attractive because it converges rapidly from any sufficient good initial guess (Dembo et al, 1982), (Kelley, 1995). However, the implementation of Newton's method involves some considerations: determining steps of Newton's method requires the solution of linear systems at each stage and exact solutions can be too expensive if the number of unknowns is large. In addition, the computational effort spent to find exact solutions for the linearized systems may not be justified when the nonlinear iterates are far from the solution. Therefore, it seems reasonable to use an iterative method (Barret et al, 1994), such as BiCGSTAB or GMRES, to solve these linear systems only approximately.

Paper accepted September, 2004. Technical Editor: Aristeu Silveira Neto.
The inexact Newton method associated with a proper iterative Krylov solver, presents an appropriated framework to solve nonlinear systems, offering a trade-off between accuracy and amount of computational effort spent per iteration. For a proper mathematical description of the inexact Newton method we refer to Kelley (1995). It is often necessary to increase the robustness of the inexact Newton method adding some globalization procedure (Kelley, 1995, Pernice et al, 1998).

In the context of the SUPG/PSPG formulation for the steady incompressible Navier-Stokes equations coupled with heat and mass transfer, the work of Shadid et al (1997) investigated in depth the behavior of the inexact Newton method with backtracking. They have shown its computational efficiency and robustness. However, they pointed out that globalization by backtracking may be less robust when high accuracy is required at each linear solve than when less accuracy is required. A mathematical explanation for this behavior is provided in Tuminaro et al (2002). In the works of Shadid et al (1997) and Tuminaro et al (2002) the Jacobian was evaluated by a mixed method. Contributions emanating from the Galerkin term were computed analytically, whereas contributions from the stabilization terms were evaluated by numerical differentiation.

In this work, we evaluate the effectiveness of some Newton-type methods dealing with problems involving steady incompressible fluid flows. We also investigate the influence of the Jacobian form described by Tezduyar (1999). This form is based on Taylor's expansions of the nonlinear terms and presents an alternative and simple way to implement the tangent matrix employed by inexact Newton-type methods. The Krylov-subspace iterative driver of the Newton-type algorithms is a nodal-block diagonal preconditioned element-by-element GMRES solver. The test problems are the wellknown driven cavity flow and flow over a backward facing step.

The remainder of this paper is organized as follows. The governing equations and the SUPG/PSPG finite element formulation are stated in Section 2. In Section 3 we present the inexact Newton and inexact successive substitution methods, showing how to choose the parameter used to force the residual to be suitably small, the backtracking strategy and the evaluation of the approximate Jacobian. In Section 4 parametric studies are presented for two test problems, accessing performance and accuracy of the nonlinear solution methods. The paper ends with a summary of our main conclusions. 


\section{Governing Equations and Finite Element Formulation}

Let $\Omega \subset \Re^{n_{s d}}$ be the spatial domain, where $n_{s d}$ is the number of space dimensions. Let $\Gamma=\Gamma_{g} \cup \Gamma_{h}$ denote the boundary of $\Omega$. We consider the following velocity-pressure formulation of the NavierStokes equations governing steady incompressible flows,

$$
\begin{array}{ll}
\rho(\mathbf{u} \cdot \boldsymbol{\nabla u}-\mathbf{f})-\boldsymbol{\nabla} \cdot \boldsymbol{\sigma}=\mathbf{0} & \text { on } \Omega \\
\boldsymbol{\nabla} \cdot \mathbf{u}=\mathbf{0} & \text { on } \Omega
\end{array}
$$

where $\rho$ and $\mathbf{u}$ are the density and velocity respectively, and $\boldsymbol{\sigma}$ is the stress tensor given as

$$
\boldsymbol{\sigma}(p, \mathbf{u})=-p \mathbf{I}+2 \mu \boldsymbol{\varepsilon}(\mathbf{u}),
$$

with,

$$
\boldsymbol{\varepsilon}(\mathbf{u})=\frac{1}{2}\left[\nabla \mathbf{u}+(\nabla \mathbf{u})^{\mathrm{T}}\right]
$$

Here, $p$ and $\mu$ are the pressure and dynamic viscosity, and $\mathbf{I}$ is the identity tensor. The essential and natural boundary conditions associated with equations (1) and (2) are represented as

$$
\begin{array}{ll}
\mathbf{u}=\mathbf{g} & \text { on } \Gamma_{g} \\
\mathbf{n} \cdot \boldsymbol{\sigma}=\mathbf{h} & \text { on } \Gamma_{h} .
\end{array}
$$

Let us assume following Tezduyar (1991) that we have some suitably defined finite-dimensional trial solution and test function spaces for velocity and pressure, $S_{\mathbf{u}}^{h}, V_{\mathbf{u}}^{h}, S_{p}^{h}$ and $V_{p}^{h}=S_{p}^{h}$. The stabilized finite element formulation of equations (1) and (2) can then be written as follows: Find $\mathbf{u}^{h} \in S_{\mathbf{u}}^{h}$ and $p^{h} \in S_{p}^{h}$ such that $\forall \mathbf{w}^{h} \in V_{\mathbf{u}}^{h}$ and $\forall q^{h} \in V_{p}^{h}:$

$$
\begin{gathered}
\int_{\Omega} \mathbf{w}^{\mathrm{h}} \cdot \rho\left(\mathbf{u}^{\mathrm{h}} \cdot \nabla \mathbf{u}^{\mathrm{h}}-\mathbf{f}\right) d \Omega+\int_{\Omega} \boldsymbol{\varepsilon}\left(\mathbf{w}^{\mathrm{h}}\right): \boldsymbol{\sigma}\left(p^{\mathrm{h}}, \mathbf{u}^{\mathrm{h}}\right) d \Omega+\int_{\Omega} q^{\mathrm{h}} \boldsymbol{\nabla} \cdot \mathbf{u}^{\mathrm{h}} d \Omega \\
+\sum_{e=1}^{n_{e l}} \int_{\Omega} \tau_{S U P G} \mathbf{u}^{h} \cdot \nabla \mathbf{w}^{h} \cdot\left[\rho\left(\mathbf{u}^{h} \cdot \nabla \mathbf{u}^{h}\right)-\nabla \cdot \boldsymbol{\sigma}\left(p^{h}, \mathbf{u}^{h}\right)-\rho \mathbf{f}\right] d \Omega \\
+\sum_{e=1}^{n_{e l}} \int_{\Omega} \frac{1}{\rho} \tau_{P S P G} \nabla q^{h} \cdot\left[\rho\left(\mathbf{u}^{h} \cdot \nabla \mathbf{u}^{h}\right)-\nabla \cdot \boldsymbol{\sigma}\left(p^{h}, \mathbf{u}^{h}\right)-\rho \mathbf{f}\right] d \Omega \\
=\int_{\Gamma} \mathbf{w}^{\mathrm{h}} \cdot \mathbf{h} d \Gamma
\end{gathered}
$$

In the above equation the first three integrals on the left hand side and the right hand side integral represent terms that appear in the standard Galerkin formulation of the problem (1)-(6), while the remaining integral expressions represent the additional terms which arise from the stabilized finite element formulation of the problem. Note that the stabilization terms are evaluated as the sums of element-wise integral expressions. The first summation corresponds to the SUPG (Streamline Upwind Petrov/Galerkin) term and the second correspond to the PSPG (Pressure Stabilization Petrov/Galerkin) term. We have calculated the stabilization parameters (Tezduyar et al, 1992) as follows,

$$
\tau_{S U P G}=\tau_{P S P G}=\left[\left(\frac{2\left\|\mathbf{u}^{h}\right\|}{h^{\#}}\right)^{2}+9\left(\frac{4 v}{\left(h^{\#}\right)^{2}}\right)^{2}\right]^{-1 / 2}
$$

Here $\mathbf{u}^{h}$ is the local velocity vector, $v$ represent the kinematic viscosity and $h^{\#}$ is the element length, defined to be equal to the diameter of the circle which is area-equivalent to the element. The spatial discretization of equation (7) leads to the following set of non-linear algebraic equations,

$$
\begin{aligned}
& \mathbf{N}(\mathbf{u})+\mathbf{N}_{\delta}(\mathbf{u})+\mathbf{K u}-\left(\mathbf{G}+\mathbf{G}_{\delta}\right) \boldsymbol{p}=\mathbf{f}_{\mathbf{u}} \\
& \mathbf{G}^{T} \mathbf{u}+\mathbf{N}_{\varphi}(\mathbf{u})+\mathbf{G}_{\varphi} \boldsymbol{p}=\mathbf{f}_{\boldsymbol{p}}
\end{aligned}
$$

where $\mathbf{u}$ is the vector of unknown nodal values of $\mathbf{u}^{h}$ and $\boldsymbol{p}$ is the vector of unknown nodal values of $p^{h}$. The non-linear vectors $\mathbf{N}(\mathbf{u}), \mathbf{N}_{\delta}(\mathbf{u})$, and $\mathbf{N}_{\varphi}(\mathbf{u})$ the matrices $\mathbf{K}, \mathbf{G}, \mathbf{G}_{\boldsymbol{\delta}}$ and $\mathbf{G}_{\varphi}$ emanate, respectively, from the convective, viscous and pressure terms. The vectors $\mathbf{f}_{\mathbf{u}}$ and $\mathbf{f}_{p}$ are due to the boundary conditions (5) and (6). The subscripts $\delta$ and $\varphi$ identify the SUPG and PSPG contributions respectively. In order to simplify the notation we denote by $\mathbf{x}=(\mathbf{u}, \boldsymbol{p})$ a vector of nodal variables comprising both nodal velocities and pressures, thus, equation (9) can be written as,

$$
\mathbf{F}(\mathbf{x})=\mathbf{0}
$$

where $\mathbf{F}(\mathbf{x})$ represents a nonlinear vector function.

For Reynolds numbers much greater than unity the nonlinear character of the equations becomes dominant, making the choice of the solution algorithm, especially with respect to its convergence and efficiency, a key issue. The search for a suitable nonlinear solution method is complicated by the existence of several procedures and their variants. In the following section we present the nonlinear solution strategies based on the Newton-type methods which are evaluated in this work.

\section{Nonlinear Solution Procedures}

Consider the nonlinear problem arising from the discretization of the fluid flow equations described by equation (10). We assume that $\mathbf{F}$ is continuously differentiable in $\Re^{n_{s d}}$ and denote its Jacobian matrix by $\mathbf{F}^{\prime} \in \mathfrak{R}^{n_{s d}}$. The Newton's method is a classical algorithm for solving equation (10) and can be enunciated as: Given an initial guess $\mathbf{x}_{0}$, we compute a sequence of steps $\mathbf{s}_{k}$ and iterates $\mathbf{x}_{k}$ as follows,

\section{Algorithm N}

FOR $k=0$ STEP 1 UNTIL "Convergence" DO:

$$
\begin{gathered}
\text { Solve } \mathbf{F}^{\prime}\left(\mathbf{x}_{k}\right) \mathbf{s}_{k}=-\mathbf{F}\left(\mathbf{x}_{k}\right) \\
\text { Set } \mathbf{x}_{k+1}=\mathbf{x}_{k}+\mathbf{s}_{k}
\end{gathered}
$$

Newton's method is attractive because it converges rapidly from any sufficiently good initial guess (see Dembo et al, 1982). However, one drawback of Newton's method is the need to solve the Newton equations (11) at each stage. Computing the exact solution using a direct method can be expensive if the number of 
unknowns is large and may not be justified when $\mathbf{x}_{k}$ is far from a solution. Thus, one might prefer to compute some approximate solution, leading to the following algorithm:

\section{Algorithm In}

FOR $k=0$ STEP 1 UNTIL "Convergence" DO:

FIND some $\eta_{k} \in[0,1)$ AND $\mathbf{s}_{k}$ THAT SATISFY

$$
\begin{gathered}
\left\|\mathbf{F}\left(\mathbf{x}_{k}\right)+\mathbf{F}^{\prime}\left(\mathbf{x}_{k}\right) \mathbf{s}_{k}\right\| \leq \eta_{k}\left\|\mathbf{F}\left(\mathbf{x}_{k}\right)\right\| \\
\text { Set } \mathbf{x}_{k+1}=\mathbf{x}_{k}+\mathbf{s}_{k}
\end{gathered}
$$

for some $\eta_{k} \in[0,1)$, where $\|\bullet\|$ is a norm of choice. This formulation naturally allows the use of an iterative solver: one first chooses $\eta_{k}$ and then applies the iterative solver to equation (11) until a $\mathbf{s}_{k}$ is determined for which the residual norm satisfies equation (12) In this context $\eta_{k}$ is often called the forcing term, since its role is to force the residual of (11) to be suitably small. This term can be specified in several ways (see, Eisenstat \& Walker, 1996, Shadid et $a l, 1997$, Pernice et al, 1998) to enhance efficiency and convergence and will be treated in the following section below. In our implementation we have used the nodal block-diagonal preconditioned GMRES $(m)$ method to solve the Newton equations (11), where $m$ represents the number of basis vectors used by GMRES algorithm (Saad, 2003).

A particularly simple scheme for solving the nonlinear system of equations (10) is a fixed point iteration procedure also known as the successive substitution, the Picard iteration, functional iteration or successive iteration. Note in the algorithms above that if we do not build the Jacobian matrix in equations (11) and (12) and the solution of previous iterations were reused, we have a successive substitution (SS) method. In this work, we have evaluated the efficiency of Newton and successive substitution methods and their inexact versions. We may also define a mixed strategy combining $\mathbf{S S}$ and $\mathbf{N}$ (or ISS and IN) iterations, to improve performance, as discussed in the following. In this strategy the Jacobian evaluation is enabled after $k$ successive substitutions. Thus, we have labeled the mixed strategy as $\boldsymbol{k}-\mathbf{S S}+\mathbf{N}$ or as $\boldsymbol{k}$-ISS+IN in the case of its inexact counterpart.

\section{Forcing Term}

We have implemented the forcing term as a variation of the choice from Eisenstat \& Walker (1996) that tends to minimize oversolving while giving fast asymptotic convergence to a solution of (10). Oversolving means that the linear equation for the Newton step is solved to a precision far beyond what is needed to correct the nonlinear iteration. Kelley (1995) considered the following measure of the degree to which the nonlinear iteration approximates the solution,

$$
\eta_{k}^{a}=\gamma\left\|\mathbf{F}\left(\mathbf{x}_{k}\right)\right\|^{2} /\left\|\mathbf{F}\left(\mathbf{x}_{k-1}\right)\right\|^{2}
$$

where $\gamma \in[0,1)$ is a parameter. In order to specify the choice at $k=0$ and bound the sequence away from 1 we set

$$
\eta_{k}^{b}= \begin{cases}\eta_{\max } & k=0, \\ \min \left(\eta_{\max }, \eta_{k}^{a}\right) & k>0 .\end{cases}
$$

Here the parameter $\eta_{\max }$ is an upper limit of the sequence $\left\{\eta_{k}^{a}\right\}$. We have chosen $\gamma=0.9$ according to Eisenstat \& Walker (1996) and adopted $\eta_{\max }=0.9,0.5,0.1$ arbitrarily in our tests.

It may happen that $\eta_{k}^{b}$ is small for one or more iterations while $\mathbf{x}_{k}$ is still far from the solution. A method of safeguarding against this possibility was suggested by Eisenstat \& Walker (1996) to avoid volatile decreases in $\eta_{k}$. The idea is that if $\eta_{k-1}$ is sufficiently large we do not let $\eta_{k}$ decrease by much more than a factor of $\eta_{k-1}$, that is:

$$
\eta_{k}^{c}= \begin{cases}\eta_{\max } & k=0, \\ \min \left(\eta_{\max }, \eta_{k}^{a}\right) & k>0, \eta_{k-1}^{2}<0.1, \\ \min \left(\eta_{\max }, \max \left(\eta_{k}^{a}, \eta_{k-1}^{2}\right)\right) & k>0, \eta_{k-1}^{2}>0.1,\end{cases}
$$

The constant 0.1 is arbitrary. According to Kelley (1995) the safeguarding does improve the performance of the iteration.

There is a chance that the final iterate will reduce $\|\mathbf{F}\|$ far beyond the desired level, consequently the cost of the solution of the linear equation for the last step will be higher than really needed. This oversolving in the final step can be controlled comparing the norm of the current nonlinear residual to the nonlinear norm at which the iteration would terminate

$$
\tau_{N L}=\tau_{\text {res }}\left\|\mathbf{F}_{0}\right\|
$$

and bounding $\eta_{k}$ by a constant multiple of $\tau_{N L} /\left\|\mathbf{F}\left(\mathbf{x}_{k}\right)\right\|$. We have used the choice proposed by Kelley (1995)

$$
\eta_{k}=\min \left(\eta_{\max }, \max \left(\eta_{k}^{c}, 0.5 \tau_{N L} /\left\|\mathbf{F}\left(\mathbf{x}_{k}\right)\right\|\right)\right)
$$

where $\tau_{N L}$ represent the nonlinear tolerance.

\section{Backtracking Procedures}

The backtracking procedures are employed, in the context of nonlinear methods, to improve the convergence capability for any initial guess (Eisenstat \& Walker, 1994). These procedures, also known as line searches or globalization procedures are based on trying to recover the convergence of a nonlinear method performing reductions on a Newton step according to the evolution of nonlinear residual. There are several forms to impose these step reductions and a constant reduction is often employed successfully, but sometimes disturbing the nonlinear convergence. In this work we employ the Armijo rule as described in Kelley (1995). This rule tries to compute a sufficient Newton step reduction without disturbing the nonlinear convergence, according to a residual decreasing function (see Kelley, 1995). Thus we may describe the Armijo rule by the procedures below.

After rejecting $j$ step reductions we have the following sequence:

$$
\left\|\mathbf{F}\left(\mathbf{x}_{k}\right)\right\|_{2},\left\|\mathbf{F}\left(\mathbf{x}_{k}+\lambda_{1} \mathbf{s}_{k}\right)\right\|_{2}, \ldots\left\|\mathbf{F}\left(\mathbf{s}_{k}+\lambda_{j-1} \mathbf{s}_{k}\right)\right\|_{2}
$$

where $\lambda$ is the reduction factor that we are looking for. Note that the sequence above can be modeled by the scalar function, 


$$
f(\lambda)=\left\|\mathbf{F}\left(\mathbf{x}_{k}+\lambda \mathbf{s}_{k}\right)\right\|_{2} .
$$

The minimal of the function (19) can be used for computing the next step reduction. According to Kelley (1995), we have used a third order polynomial to compute the step reduction. This procedure can be summarized as: After reject $\lambda_{c}$ and build the polynomial model we compute the minimal of $\lambda_{t}$ analytically and the following adjustment is performed,

$$
\lambda_{j+1}= \begin{cases}\sigma_{0} \lambda_{c} & \text { if } \lambda_{t}<\sigma_{0} \lambda_{c} \\ \sigma_{1} \lambda_{c} & \text { if } \lambda_{t}>\sigma_{1} \lambda_{c} \\ \lambda_{t} & \text { otherwise }\end{cases}
$$

In a 3-point parabolic model, the procedure for evaluating $f(0)$ and $f(1)$ is performed by the following steps. If the step for $\lambda=1$ was rejected, adjust $\lambda=\sigma_{1}$ and try again. After the second step rejection, we have the following values to construct the 3-point parabolic model,

$$
f(0), f\left(\lambda_{c}\right) \text { and } f\left(\lambda_{j}\right)
$$

where $\lambda_{c}$ and $\lambda_{j}$ are the values of $\lambda$ most recently rejected. The interpolation polynomial of $f$ at the points $0, \lambda_{\mathrm{c}}, \lambda_{j}$ is,

$$
\mathrm{p}(\lambda)=f(0)+\frac{\lambda}{\lambda-\lambda_{j}}\left(\frac{\left(\lambda-\lambda_{j}\right)\left(f\left(\lambda_{c}\right)-f(0)\right)}{\lambda_{c}}+\frac{\left(\lambda_{c}-\lambda\right)\left(f\left(\lambda_{j}\right)-f(0)\right)}{\lambda_{j}}\right)
$$

We must evaluate two possibilities for the curvature of polynomial in equation (22). Thus we have,

$$
\mathrm{p}^{\prime \prime}(0)=\frac{2 \lambda_{c} \lambda_{j}}{\lambda_{c}-\lambda_{j}}\left(\lambda_{j}\left(f\left(\lambda_{c}\right)-f(0)\right)-\lambda_{c}\left(f\left(\lambda_{j}\right)-f(0)\right)\right),
$$

and

$$
\lambda_{t}=-\frac{\mathrm{p}^{\prime}(0)}{\mathrm{p}^{\prime \prime}(0)}
$$

If the curvature of $\mathrm{p}$ is positive, set $\lambda_{t}$ to the minimal of $\mathrm{p}$ and compute $\lambda_{j+1}$ by equation (20); otherwise $\mathrm{p}^{\prime \prime}(0) \leq 0$, set $\lambda_{j+1}$ as the minimal of $\mathrm{p}$ in the interval $\left[\sigma_{0} \lambda, \sigma_{1} \lambda\right]$ or reject the parabolic model and set $\lambda_{j+1}$ as $\sigma_{0} \lambda$ or $\sigma_{1} \lambda$. In this work we adopted the second strategy, setting $\lambda_{j+1}=\sigma_{1} \lambda$.

\section{Jacobian Matrix Evaluation}

To form the Jacobian $\mathbf{F}^{\prime}$ required by Newton-type methods we use a numerical approximation described by Tezduyar (1999). Consider the following Taylor expansion for the nonlinear convective term emanating from the Galerkin formulation:

$$
\mathbf{N}(\mathbf{u}+\Delta \mathbf{u})=\mathbf{N}(\mathbf{u})+\frac{\partial \mathbf{N}}{\partial \mathbf{u}} \Delta \mathbf{u}+\ldots
$$

where $\mathbf{\Delta} \mathbf{u}$ is the velocity increment. Discarding the high order terms and omitting the integral symbols we arrive to the following approximation,

$$
\begin{array}{r}
\rho(\mathbf{u}+\Delta \mathbf{u}) \cdot \boldsymbol{\nabla}(\mathbf{u}+\Delta \mathbf{u}) \cong \rho(\mathbf{u} \cdot \boldsymbol{\nabla}) \mathbf{u} \\
+\rho(\mathbf{u} \cdot \boldsymbol{\nabla}) \Delta \mathbf{u}+\rho(\boldsymbol{\Delta} \mathbf{u} \cdot \boldsymbol{\nabla}) \mathbf{u}
\end{array}
$$

Note that the first term in the right hand side of equation (26) is the corresponding residual vector and the remaining terms represent the numerical approximation of $\partial \mathrm{N} / \partial \mathbf{u}$. If we apply similar derivations to $\mathbf{N}_{\delta}(\mathbf{u})$ and $\mathbf{N}_{\boldsymbol{\varphi}}(\mathbf{u})$ we obtain,

$$
\begin{aligned}
& \tau_{S U P G} \rho[(\mathbf{u}+\Delta \mathbf{u}) \cdot \nabla] \mathbf{w} \cdot[(\mathbf{u}+\Delta \mathbf{u}) \cdot \nabla](\mathbf{u}+\Delta \mathbf{u}) \cong \\
& \tau_{S U P G} \rho(\mathbf{u} \cdot \nabla) \mathbf{w} \cdot(\mathbf{u} \cdot \nabla) \mathbf{u}+\tau_{S U P G} \rho(\mathbf{u} \cdot \nabla) \mathbf{w} \cdot(\Delta \mathbf{u} \cdot \nabla) \mathbf{u} \\
&+\tau_{S U P G} \rho(\mathbf{u} \cdot \nabla) \mathbf{w} \cdot(\mathbf{u} \cdot \nabla) \Delta \mathbf{u}+\tau_{S U P G} \rho(\Delta \mathbf{u} \cdot \nabla) \mathbf{w} \cdot(\mathbf{u} \cdot \nabla) \mathbf{u}
\end{aligned}
$$

and

$$
\begin{aligned}
\tau_{P S P G} \boldsymbol{\nabla} \boldsymbol{q} \cdot[(\mathbf{u}+\boldsymbol{\Delta u}) \cdot \boldsymbol{\nabla}](\mathbf{u}+\Delta \mathbf{u}) \cong \\
\quad \tau_{P S P G} \boldsymbol{\nabla} \boldsymbol{q} \cdot(\mathbf{u} \cdot \boldsymbol{\nabla}) \mathbf{u}+\tau_{P S P G} \boldsymbol{\nabla} \boldsymbol{q} \cdot(\boldsymbol{\Delta} \mathbf{u} \cdot \boldsymbol{\nabla}) \mathbf{u}+\tau_{P S P G} \boldsymbol{\nabla} \boldsymbol{q} \cdot(\mathbf{u} \cdot \boldsymbol{\nabla}) \boldsymbol{\Delta} \mathbf{u}
\end{aligned}
$$

where again the first terms in the right hand side of equations (27) and (28) are the SUPG and PSPG contributions to the residual vector and the remaining terms define the approximations of $\partial \mathbf{N}_{\delta} / \partial \mathbf{u}$ and $\partial \mathbf{N}_{\varphi} / \partial \mathbf{u}$.

We do not assemble and store the resulting Jacobian, but rather we compute its action in the matrix-vector product needed in GMRES from element contributions, that is,

$$
\mathbf{F}^{\prime} \mathbf{x}=\stackrel{\mathbf{A}}{n=1}_{\mathbf{F}_{e}^{\prime} \mathbf{x}_{e}}
$$

where $\mathbf{A}$ is the standard finite element assembly operator (Hughes, 2000), $\mathbf{F}_{e}^{\prime}$ is the e-th contribution for the global Jacobian and $\mathbf{x}_{\mathrm{e}}$ is the restriction of a general search direction to the element degrees of freedom. A similar strategy is employed in the successive substitution methods.

\section{Test Problems}

In this section we present the results obtained with the formulation described in the previous sections applied to two classical CFD problems. The first example consists of the lid driven cavity flow and the second is the flow over a backward facing step. For both examples we have tested the nonlinear algorithms proposed at Reynolds numbers 100, 500 and 1000. The numerical procedure considers a fully coupled $\mathbf{u}-p$ version of stabilized formulation using linear triangular elements. The computations were halted when the following criteria were met: $\|\mathbf{F}\| /\left\|\mathbf{F}_{0}\right\| \leq 10^{-3}$ and $\|\mathbf{s}\| /\|\mathbf{x}\| \leq 10^{-3}$ or the total number of nonlinear iterations exceeds 1000 .

\section{Lid Driven Cavity flow}

The two-dimensional flow in a driven cavity which the top wall moves with a uniform velocity has been used rather extensively as a validation test case by many authors in the last years (see, Ghia et $a l, 1982)$. In this problem the Reynolds number is based on the size 
of the cavity, the flow velocity on the lid and fluid viscosity. The problem domain and mesh with 1,681 nodes and 3,200 elements are presented in Figs. 1a and $1 \mathrm{~b}$ respectively.

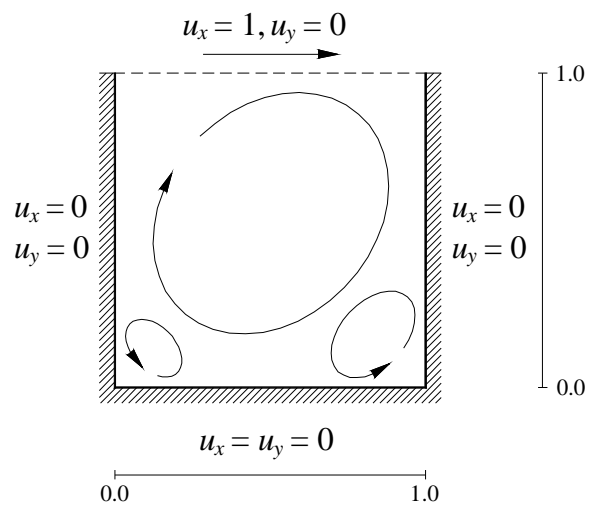

(a)

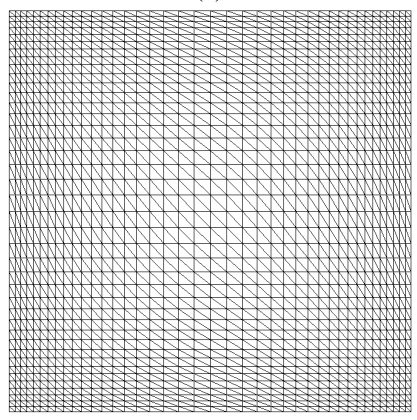

(b)

Figure 1. Two-dimensional lid-driven cavity problem - (a) Domain Problem, (b) Finite element mesh (1,681 nodes and 3,200 elements).

Table 1 shows the results for tests with different nonlinear strategies to solve the lid driven cavity flow at Reynolds number 100, 500 and 1000. In all tests we employed GMRES(45) with nodal block-diagonal preconditioning to solve the linear problem. For the classical Newton-type methods the linear solver tolerance was set to $10^{-6}$. For the inexact methods we have tested $0.1,0.5$ and 0.9 as the maximum linear solver tolerance, $\eta_{\max }$. For each Table presented in this section, the first column lists the nonlinear method employed, where SS and ISS- $\eta$ labels represent the Successive Substitution and Inexact Successive Substitution methods respectively and $\eta$ is the maximum linear solver tolerance adopted by the inexact method. The second column shows the number of linear iterations (\# LI) performed by GMRES. The third column gives the number of nonlinear iterations (\# NLI) performed by each method. The fourth column presents the time spent in the solution process and the Euclidean solution norms $\left(\|\bullet\|_{2}\right)$ were presented on the three last columns as solution quality indicators. Some Tables present two additional columns with the label (INFO) comprising warning messages or information about the solution process and (BCT) with the number of backtracking steps performed.
Table 1. Performance of Successive Substitution and Inexact Successive Substitution. Influence of the maximum linear solver tolerance. GMRES(45), $R e=100,500$ and 1000.

\begin{tabular}{ccccccc} 
& $\# \mathbf{L I}$ & $\# \mathbf{N L I}$ & $\begin{array}{c}\text { Time } \\
(\mathbf{s})\end{array}$ & $\left\|\mathbf{u}_{\boldsymbol{x}}\right\|_{\mathbf{2}}$ & $\left\|\mathbf{u}_{\boldsymbol{y}}\right\|_{\mathbf{2}}$ & $\|\boldsymbol{p}\|_{\mathbf{2}}$ \\
\hline $\boldsymbol{R} \boldsymbol{e}=\mathbf{1 0 0}$ & & & & & & \\
SS & 8668 & 5 & 38.0 & 10.6873 & 5.6228 & 5.4948 \\
ISS-0.1 & 1388 & 7 & 6.08 & 10.6873 & 5.6210 & 5.4783 \\
ISS-0.5 & 1174 & 13 & 5.15 & 10.6862 & 5.6183 & 5.4748 \\
ISS-0.9 & 779 & 11 & 3.40 & 10.6826 & 5.5825 & 5.4686 \\
\hline $\boldsymbol{R} \boldsymbol{e}=\mathbf{5 0 0}$ & & & & & & \\
SS & 11678 & 7 & 50.8 & 9.6971 & 5.7740 & 2.2638 \\
ISS-0.1 & 1831 & 14 & 8.05 & 9.6979 & 5.7738 & 2.2163 \\
ISS-0.5 & 1142 & 23 & 5.03 & 9.6974 & 5.7687 & 2.2114 \\
ISS-0.9 & 968 & 41 & 4.28 & 9.6972 & 5.7600 & 2.2065 \\
\hline $\boldsymbol{R} \boldsymbol{e}=\mathbf{1 0 0 0}$ & & & & & & \\
SS & 19850 & 11 & 86.3 & 9.0300 & 5.2626 & 1.5739 \\
ISS-0.1 & 1685 & 14 & 7.37 & 9.0318 & 5.2653 & 1.5234 \\
ISS-0.5 & 1199 & 28 & 5.27 & 9.0345 & 5.2681 & 1.5162 \\
ISS-0.9 & 905 & 39 & 4.01 & 9.0342 & 5.2689 & 1.5182 \\
\hline
\end{tabular}

We can see in Table 1 that although the classical SS method requires less nonlinear iterations, it needed more GMRES iterations and thus, it is slower than the inexact method. We also observe that increasing the Reynolds number the solution process becomes more difficult.

Table 2 presents a performance comparison among the Newtontype implementations described in previous sections. These Newton-type methods differ by the form on which the Jacobian matrix evaluation is performed in the linearized problem. In the ISS method the nonlinear derivatives are not evaluated during the solution process. In the inexact Newton method (IN) the approximated Jacobian matrix is built and evaluated using the expressions in equations (26) to (28) from the start to the end of the nonlinear solution. We may also use a mixed solution strategy to circumvent initialization problems observed in some problems. In this strategy we enable the approximate Jacobian evaluation after $k$ successive substitutions, thus this method will be labeled as $k$ ISS+IN. We have adopted arbitrarily in our tests $k=5$ and $\eta_{\max }=0.1$.

Table 2. Performance of the Newton-type methods. GMRES(45), $\eta_{\max }=0.1$, $R e=100,500$ and 1000 .

\begin{tabular}{ccccccc} 
& $\# \mathbf{L I}$ & $\# \mathbf{N L I}$ & $\begin{array}{c}\text { Time } \\
(\mathbf{s})\end{array}$ & $\left\|\mathbf{u}_{x}\right\|_{2}$ & $\left\|\mathbf{u}_{y}\right\|_{2}$ & $\|\boldsymbol{p}\|_{2}$ \\
\hline $\boldsymbol{R} \boldsymbol{e}=\mathbf{1 0 0}$ & & & & & & \\
ISS & 1388 & 7 & 6.05 & 10.6873 & 5.6210 & 5.4783 \\
IN & 1400 & 5 & 6.12 & 10.6871 & 5.6224 & 5.4794 \\
$5-$ ISS+IN & 1543 & 7 & 6.67 & 10.6871 & 5.6224 & 5.4787 \\
\hline $\boldsymbol{R} \boldsymbol{e}=\mathbf{5 0 0}$ & & & & & & \\
ISS & 1832 & 14 & 8.00 & 9.6979 & 5.7738 & 2.2163 \\
IN & 1952 & 10 & 8.54 & 9.6970 & 5.7739 & 2.7762 \\
$5-$ ISS+IN & 1405 & 9 & 6.05 & 9.6970 & 5.7739 & 2.2162 \\
\hline $\boldsymbol{R} \boldsymbol{e}=\mathbf{1 0 0 0}$ & & & & & & \\
ISS & 1685 & 14 & 7.30 & 9.0318 & 5.2653 & 1.5234 \\
IN & 42818 & 12 & 186.0 & 9.0306 & 5.2636 & 1.5995 \\
$5-$ ISS+IN & 1488 & 10 & 6.39 & 9.0308 & 5.2639 & 1.5230 \\
\hline
\end{tabular}

Table 2 shows that the inexact successive substitution method executed more nonlinear iterations, however, these iterations spent less time at all Reynolds numbers than the inexact Newton method. Note that the mixed method (5-ISS+IN) also presented good performance, spending less nonlinear iterations and time than the other methods for Reynolds 500 and 1000. We also note discrepancies among the pressure norms for IN cases at Reynolds numbers 500 and 1000 . Clearly the numerically approximated Jacobian deteriorates the GMRES performance. This behavior may 
be associated to an ill-conditioning of the resulting numerically approximated Jacobian. Figs 2a to 2c show the residual decay per iteration for each strategy in Table 2.

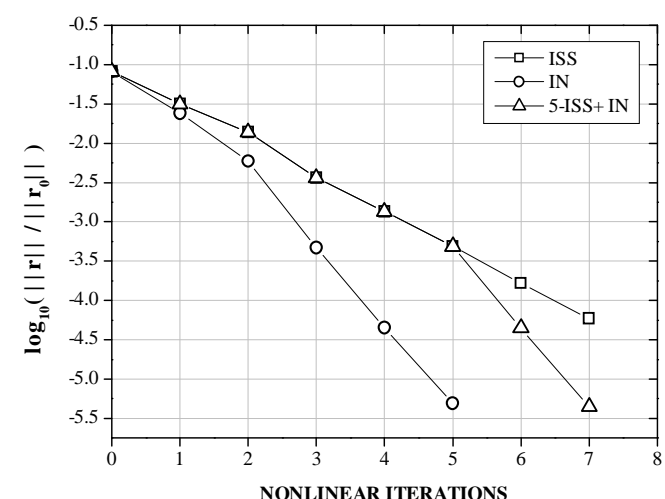

(a) Reynolds 100

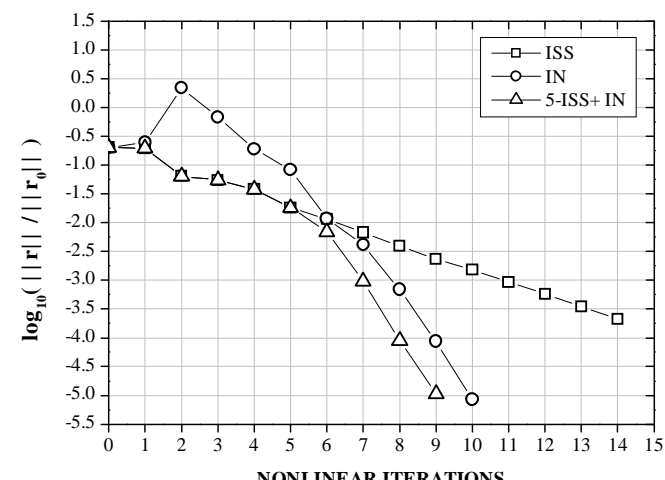

NONLINEAR ITERATIONS

(b) Reynolds 500

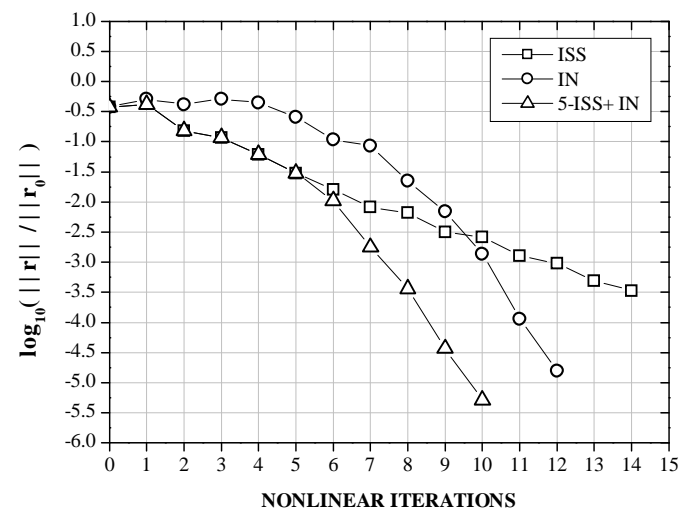

(c) Reynolds 1000

Figure 2. Convergence history - Influence of numerical Jacobian evaluation in Newton-type methods.

We can note in these Figures that for increasing Reynolds numbers convergence deteriorates. These Figures also show that convergence is faster for the methods based in the numerically approximated Jacobian evaluations than for the successive substitution methods.

In Table 3 we present the results of inexact nonlinear methods with backtracking enabled. Note that backtracking was invoked only for $\mathrm{Re}=500$ and 1000 . It was particularly important for recovering accuracy and reducing processing times of the IN solutions.
Table 3. Performance of inexact methods with globalization (backtracking) procedures. GMRES(45), $\eta_{\max }=0.1, R e=100,500$ and 1000.

\begin{tabular}{cccccccc} 
& \# LI & $\begin{array}{c}\# \\
\text { NLI }\end{array}$ & $\begin{array}{c}\text { Time } \\
(\mathbf{s e c})\end{array}$ & $\# \mathbf{B C T}$ & $\left\|\mathbf{u}_{x}\right\|_{2}$ & $\left\|\mathbf{u}_{\boldsymbol{y}}\right\|_{2}$ & $\|\boldsymbol{p}\|_{2}$ \\
\hline $\boldsymbol{R} \boldsymbol{e}=\mathbf{1 0 0}$ & & & & & & & \\
ISS & 1388 & 7 & 6.0 & 0 & 10.687 & 5.621 & 5.478 \\
IN & 1401 & 5 & 6.1 & 0 & 10.687 & 5.622 & 5.479 \\
5 -ISS+IN & 1543 & 7 & 6.7 & 0 & 10.687 & 5.622 & 5.479 \\
\hline $\boldsymbol{R} \boldsymbol{e}=\mathbf{5 0 0}$ & & & & & & & \\
ISS & 1831 & 14 & 7.9 & 0 & 9.698 & 5.774 & 2.216 \\
IN & 1478 & 7 & 6.4 & 2 & 9.697 & 5.774 & 2.216 \\
5-ISS+IN & 1405 & 9 & 6.1 & 0 & 9.697 & 5.774 & 2.216 \\
\hline $\boldsymbol{R} \boldsymbol{e}=$ & & & & & & & \\
$\mathbf{1 0 0 0}$ & & & & & & & \\
ISS & 1783 & 14 & 7.8 & 1 & 9.032 & 5.266 & 1.514 \\
IN & 1641 & 9 & 7.1 & 3 & 9.031 & 5.263 & 1.524 \\
5-ISS+IN & 1339 & 9 & 5.8 & 1 & 9.031 & 5.265 & 1.513 \\
\hline
\end{tabular}

Figures 3 and 4 show the results obtained with the nonlinear methods for Reynolds numbers 100, 500 and 1000. These results were obtained with the inexact successive substitution method. Note in these Figures the vortex displacement for the center of the square for increasing Reynolds numbers.

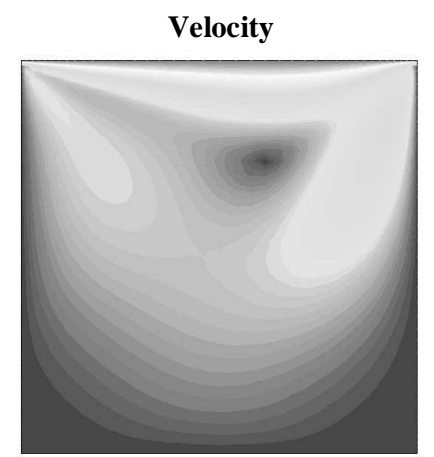

(a) Reynolds 100

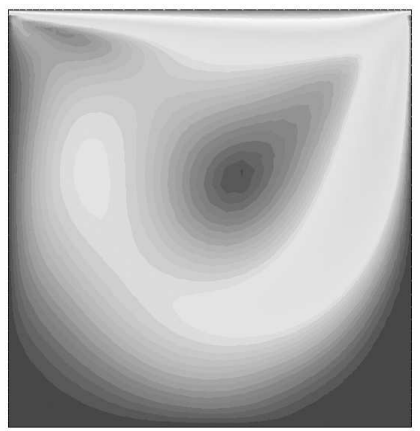

(b) Reynolds 500

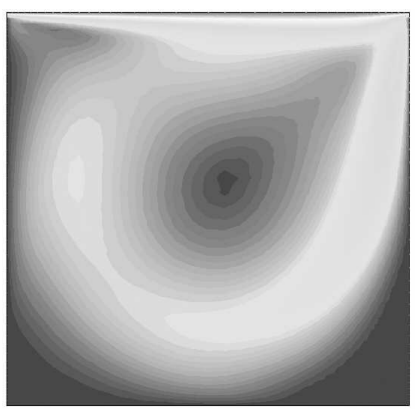

(c) Reynolds 1000

Figure 3. Steady-state solution for the lid-driven cavity flow. 


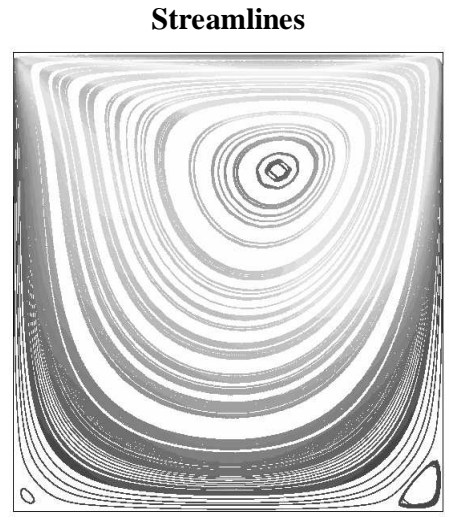

(a) Reynolds 100

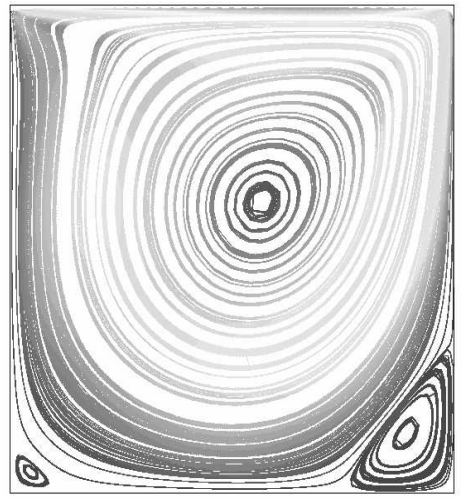

(b) Reynolds 500

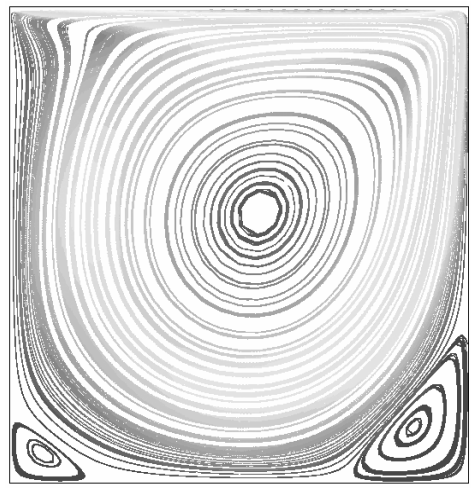

(c) Reynolds 1000

Figure 4. Steady-state solution for the lid-driven cavity flow.

\section{Flow Over a Backward Facing Step}

As a second example we consider the flow over a backward facing step, which also has become popular as a test problem for flow simulation codes. It consists of a fluid flowing into a straight channel which abruptly widens on one side. Numerical results obtained using a wide range of methods can be found in Gartling (1990). When the fluid flows downstream, it produces a recirculation zone on the lower channel wall, and for sufficiently high Reynolds it also produces a recirculation zone farther downstream on the upper wall. The finite element mesh with 1,800 elements and 1,021 nodes, boundary conditions and problem domain are present in Fig. 5a-b. Note that the Fig. 5a shows only the part of the computational domain that contains all the essential features.

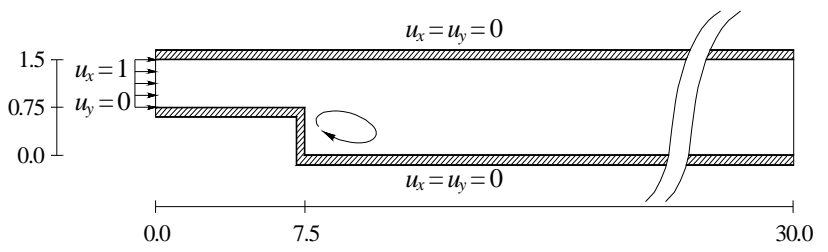

(a)

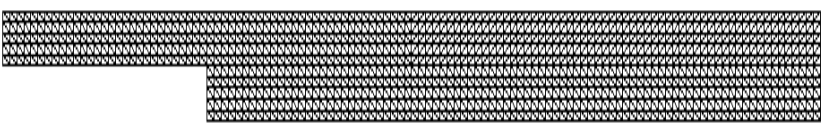

(b)

Figure 5. Flow over a backward facing step. (a) Problem domain and (b) finite element mesh (1,800 elements and 1,021 nodes).

Table 4 presents the performance results obtained for the Newton-type methods. The definitions adopted here are the same employed in the previous example. The additional column identified by $\mathbf{R M}$, means one more quality parameter. It represents the Residual Mass found for the incompressibility constraint given by equation (2).

Here again, we observe that the inexact methods needed more nonlinear iterations. As in the previous example, these iterations required less time. Observe that for Reynolds 1000, only SS and ISS- 0.1 methods were able to solve this problem for all variables. We note that the classical methods were more conservative, presenting less residual mass than the inexact methods. However, the residual mass of the converged inexact Newton solutions are of the same order of the required nonlinear accuracy. Table 5 shows the results obtained for the numerical Jacobian influence tests applied to the backward facing step problems. The tolerances and the other parameters were the same employed in the lid driven cavity flow problem.

Table 5 shows that the methods based on numerical Jacobian evaluations needed less nonlinear iterations. However, these methods were less efficient, spending more time than successive substitution methods. Also in this case the methods based on numerical Jacobian evaluations were less accurate for high Reynolds numbers, as indicated in Table 5. Figure 6 presents the residual decays for the backward facing step problems.

In Table 6 we present the results for the inexact methods with globalization procedures. Note that in this case the globalization procedures do not have any positive effect. For $\mathrm{Re}=500$ and 1000 even the ISS method was unable to solve this problem with globalization switched on. 
Table 4. Performance of Successive Substitution and Inexact Successive Substitution. Influence of the maximum tolerance choice. GMRES(45), Re = 100, 500 and 1000.

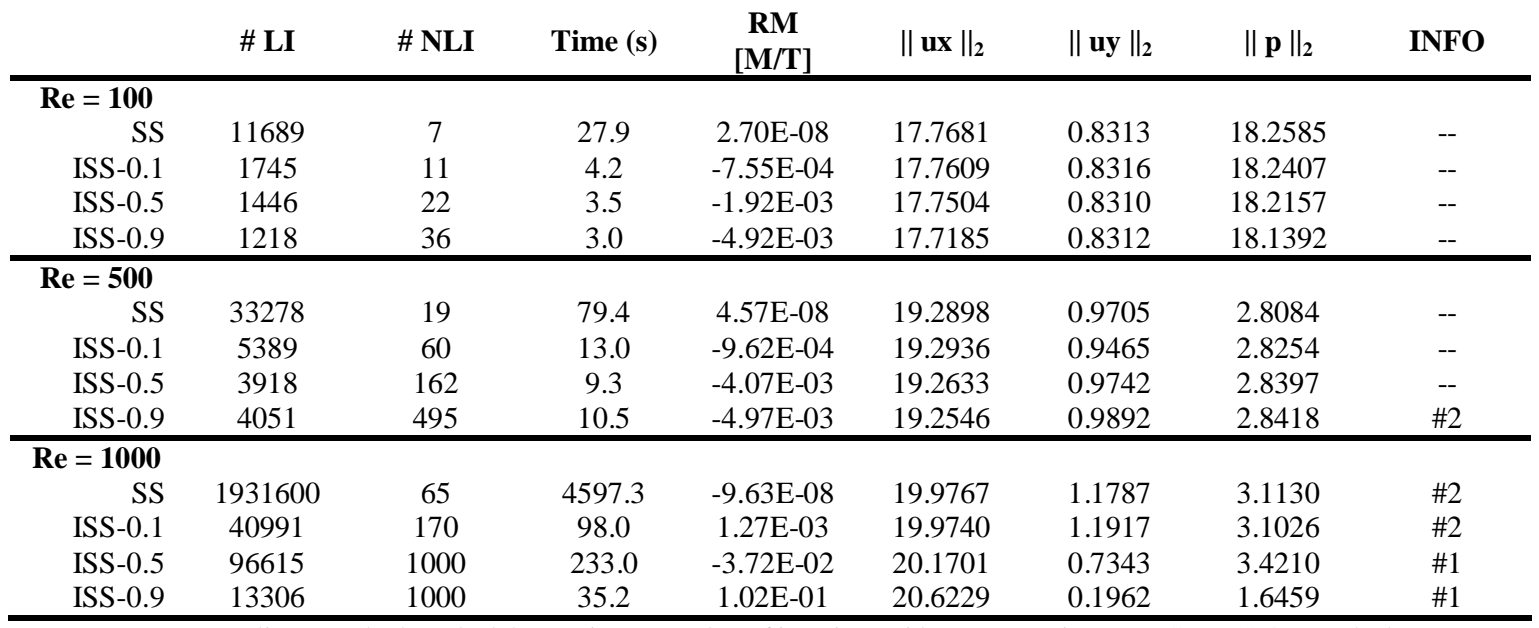

\#1 - Nonlinear method reached the maximum number of iterations without converging. \#2 - GMRES(45) reached the maximum number of iterations without converging in some nonlinear step.

Table 5. Performance of the Newton-type methods. GMRES(45), $\eta_{\max }=0.1, R e=100,500$ and 1000.

\begin{tabular}{|c|c|c|c|c|c|c|c|c|}
\hline & \# LI & \# NLI & $\begin{array}{l}\text { Time } \\
\text { (sec) }\end{array}$ & $\begin{array}{c}\mathbf{R M} \\
{[\mathrm{M} / \mathrm{T}]}\end{array}$ & $\left\|\mathbf{u}_{x}\right\|_{2}$ & $\left\|\mathbf{u}_{y}\right\|_{2}$ & $\|p\|_{2}$ & INFO \\
\hline \multicolumn{9}{|l|}{$R e=100$} \\
\hline ISS & 1745 & 11 & 4.2 & $-7.55 \mathrm{E}-04$ & 17.7609 & 0.8316 & 18.2407 & -- \\
\hline IN & 2155 & 8 & 5.1 & $-2.97 E-05$ & 17.7682 & 0.8307 & 18.2579 & $\# 2$ \\
\hline 5-ISS+IN & 1968 & 9 & 4.7 & $-8.26 \mathrm{E}-05$ & 17.7676 & 0.8307 & 18.2567 & $\# 2$ \\
\hline \multicolumn{9}{|l|}{$R e=500$} \\
\hline ISS & 5389 & 60 & 12.8 & $-9.62 \mathrm{E}-04$ & 19.2936 & 0.9465 & 2.8254 & -- \\
\hline IN & 83142 & 21 & 198.9 & $-8.75 \mathrm{E}-03$ & 19.2038 & 1.0314 & 2.8327 & $\# 4$ \\
\hline 5-ISS+IN & 95560 & 31 & 228.5 & $-1.54 \mathrm{E}-02$ & 19.1303 & 1.0861 & 2.8484 & $\# 4$ \\
\hline \multicolumn{9}{|l|}{$R e=1000$} \\
\hline ISS & 40991 & 170 & 97.9 & $1.27 \mathrm{E}-03$ & 19.9740 & 1.1917 & 3.1026 & $\# 2$ \\
\hline IN & 127384 & 28 & 298.4 & $3.96 \mathrm{E}-02$ & 20.4647 & 0.4220 & 2.7222 & \#4 \\
\hline $5-\mathrm{ISS}+\mathrm{IN}$ & 130485 & 30 & 312.2 & $7.05 \mathrm{E}-02$ & 20.5361 & 0.2206 & 2.0798 & \#4 \\
\hline
\end{tabular}

\#2 - GMRES(45) reached the maximum number of iterations without converging in some nonlinear step. \#4 nonlinear method stagnated without converging.

Table 6. Performance of inexact methods with globalization (backtracking) procedures. GMRES(45), $\eta_{\max }=0.1, \operatorname{Re}=100,500$ and 1000 .

\begin{tabular}{|c|c|c|c|c|c|c|c|c|}
\hline & \# LI & \# NLI & $\begin{array}{l}\text { Time } \\
\text { (sec) }\end{array}$ & BCT & $\left\|\mathbf{u}_{x}\right\|_{2}$ & $\left\|\mathbf{u}_{y}\right\|_{2}$ & $\|p\|_{2}$ & INFO \\
\hline \multicolumn{9}{|l|}{$\operatorname{Re}=100$} \\
\hline ISS & 1745 & 11 & 4.2 & 0 & 17.7609 & 0.8316 & 18.2407 & -- \\
\hline IN & 2084 & 8 & 5.0 & 2 & 17.7672 & 0.8307 & 18.2555 & $\# 2$ \\
\hline 5-ISS+IN & 1968 & 9 & 4.7 & 0 & 17.7676 & 0.8307 & 18.2567 & $\# 2$ \\
\hline \multicolumn{9}{|l|}{$R e=500$} \\
\hline ISS & 2321 & 22 & 5.5 & 22 & 19.4987 & 0.6019 & 2.8774 & \#3 \\
\hline IN & 62265 & 16 & 148.7 & 33 & 19.5327 & 0.6333 & 2.8968 & \#3 \\
\hline $5-\mathrm{ISS}+\mathrm{IN}$ & 62889 & 16 & 150.1 & 29 & 19.4784 & 0.7085 & 2.8936 & \#3 \\
\hline \multicolumn{9}{|l|}{$R e=1000$} \\
\hline ISS & 117232 & 35 & 280.5 & 29 & 20.3386 & 0.2414 & 2.6355 & \#3 \\
\hline IN & 34452 & 4 & 81.9 & 23 & 9.8003 & 0.3398 & 0.3897 & \#3 \\
\hline $5-\mathrm{ISS}+\mathrm{IN}$ & 23513 & 7 & 56.2 & 26 & 19.1764 & 0.6449 & 1.2578 & \#3 \\
\hline
\end{tabular}

\#2 - GMRES(45) reached the maximum number of iterations without converging in some nonlinear step.\#3 -

Backtracking failure after 20 step reductions. 


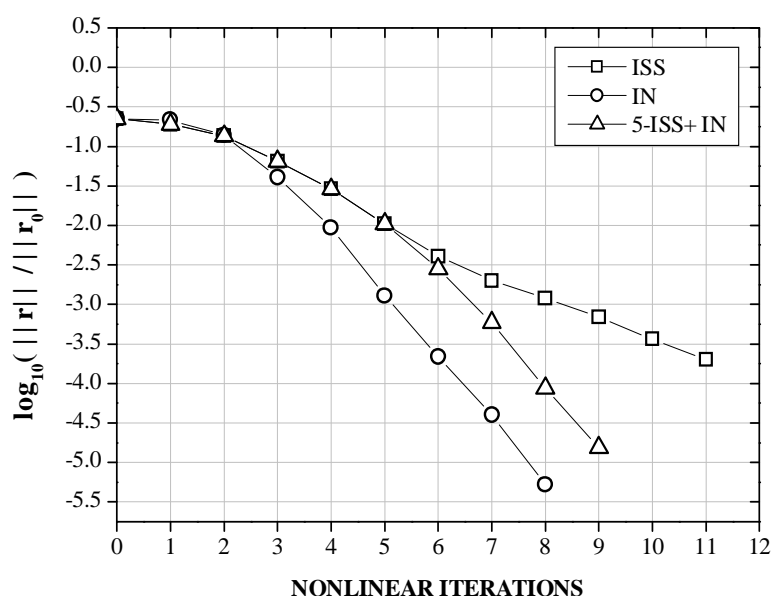

(a) Reynolds 100

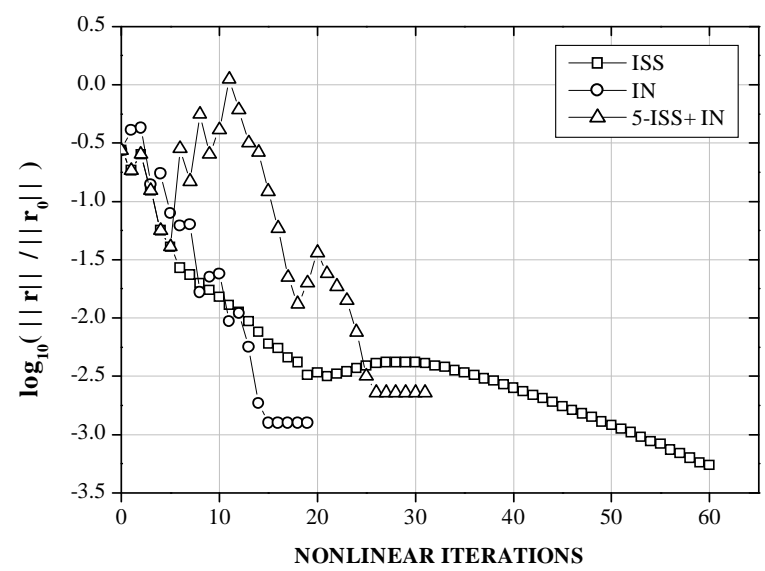

(b) Reynolds 500

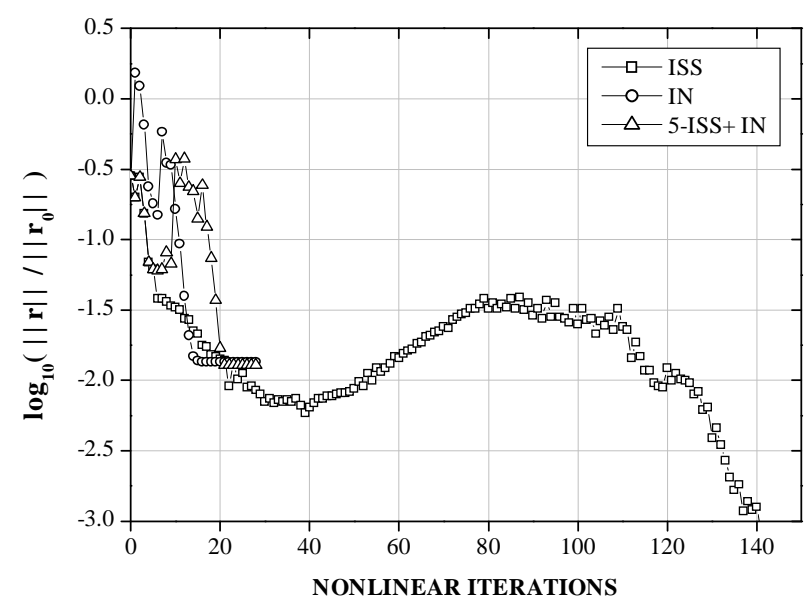

(c) Reynolds 1000

Figure 6. Convergence history - Influence of numerical Jacobian evaluation in Newton-type methods.

These Figures show an increasing difficulty to solve the backward facing step problem for high Reynolds numbers. For Reynolds 500 and 1000 the methods involving numerical approximate Jacobian stagnate without reaching convergence.
Figures 7 to 9 show the converged solutions obtained employing the strategies listed in previous Tables.

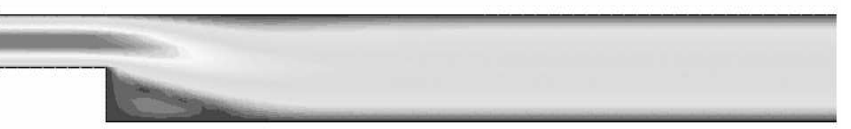

(a) Velocity

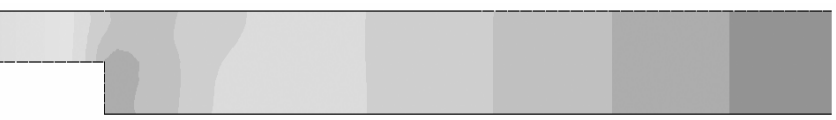

(b) Pressure

Figure 7. Steady state solution for the backward facing step flow at Reynolds number 100.

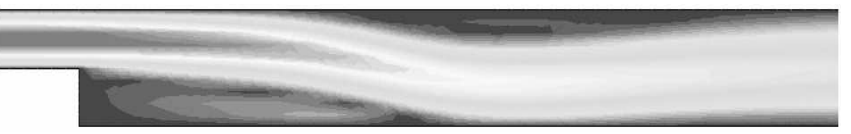

(a) Velocity

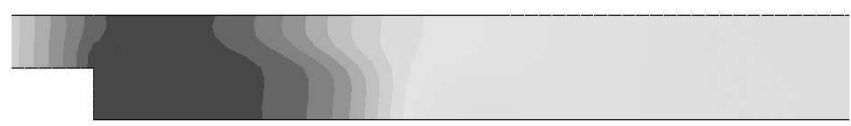

(b) Pressure

Figure 8. Steady state solution for the backward facing step flow at Reynolds number 500 .

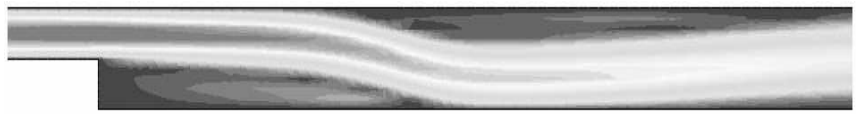

(a) Velocity

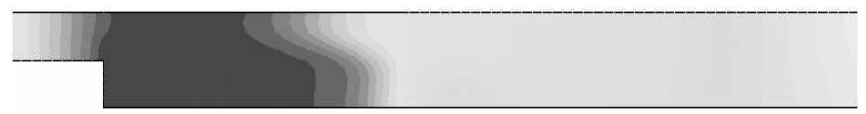

(b) Pressure

Figure 9. Steady state solution for the backward facing step flow at Reynolds number 1000 .

We can see the vortex recirculation forming on the top wall at high Reynolds numbers problems. This behavior is characteristic on backward facing step simulations.

\section{Conclusions}

In this work we compare several Newton-type algorithms to solve nonlinear problems arising from the SUPG/PSPG stabilized finite element formulation of the steady incompressible NavierStokes equations. Preconditioned GMRES is used as the linear iterative solver and the fully coupled Jacobian is numerically approximated by a truncated Taylor expansion. We introduced a mixed strategy which combines the successive substitution method with Newton's method using the numerically approximated Jacobians.

We observed that in general the inexact methods are simple to implement, fast and accurate. The numerically approximated Jacobian reduces the number of nonlinear iterations. However, the total number of GMRES iterations increases significantly, resulting in more CPU time than the corresponding successive substitution solutions. The mixed method combines the good features of both methods. In the backward facing step problem at Reynolds numbers 500 and 1000 the inexact solutions with the numerically 
approximated Jacobian needed globalization procedures to achieve a solution. This is an indication that effective solutions with this option may need more robust preconditioners than the simple nodal block-diagonal preconditioner used in this work. Our numerical results also indicate that globalization procedures must be used with care.

Sophisticated nonlinear solution methods are certainly needed for solving complex flow problems. However, they generally involve the choice of many parameters, which requires a high degree of expertise from the users. More numerical experiments are needed to access the influence of all parameters and to provide guidelines for the inexperienced user.

\section{Acknowledgements}

The authors would like to thank the financial support of the Petroleum National Agency (ANP, Brazil). The Center for Parallel Computations (NACAD) and the Laboratory of Computational Methods in Engineering (LAMCE) at the Federal University of Rio de Janeiro provided the computational resources for this research. We gratefully acknowledge Prof. T. E. Tezduyar from Rice University for his assistance with some details on the stabilized finite element formulation.

\section{References}

Aliabadi, S., Johnson, A., Abedi, J., Zellars, B., 2002, "High Performance Computing of Fluid-Structure Interactions in Hydrodynamics Applications Using Unstructured Meshes with More than One Billion Elements", Lecture Notes in Computer Science, 2552, pp. 519-533.

Barret, R., Berry, M., Chan. T.F., Demmel, J., Donato, J., Dongarra, J., Eijkhout, V., Pozo, R., Romine, C., van der Vorst, H., 1994, Templates for the Solution of Linear Systems: Building Blocks for Iterative Methods, SIAM Philadelphia;

Brooks, A. N. and Hughes, T. J. R., 1982, "Streamline Upwind/PetrovGalerkin Formulations for Convection Dominated Flows with Particular Emphasis on the Incompressible Navier-Stokes Equation", Comput. Methods Appl. Mech. Engrg. 32, pp. 199-259.

Dembo, R., S., Eisenstat, S. C. and Steihaug, T., 1982, "Inexact Newton Methods", SIAM J. Numer. Anal., 19, pp. 400-408.
Eisenstat, S. C. and Walker, H. F., 1996, "Choosing the Forcing Terms in Inexact Newton Method", SIAM. J. Sci Comput. 17-1, 16-32.

Eisenstat, S. C. and Walker, H. F., 1994, "Globally Convergent Inexact Newton Methods", SIAM J. Optim., 4, pp. 393-422.

Gartling, D. K., 1990, "A Test Problem for Outflow Boundary Conditions - Flow Over a Backward-Facing Step", Internat. J. Numer. Methods Fluids, Vol. 11, 953-967.

Guia, U., Ghia, K. and Shin, C., 1982. "High-Re Solutions for Incompressible Flow Using the Navier-Stokes Equations and a Multigrid Method", J. Comput. Phys., 48, 387-411.

Hughes, T.J.R., 2000, "The Finite Element Method: Linear Static and Dynamic Finite Element Analysis", Dover Pubns.

Hughes, T. J. R., Franca, L. P. and Balestra, M., 1986, “A New Finite Element Formulation for Computational Fluid Dynamics: V. Circumventing the Babuška-Brezzi Condition: A Stable Petrov-Galerkin Formulation of the Stokes Problem Accommodating Equal-Order Interpolations", Comput. Methods Appl. Mech. Engrg., 59, pp. 85-99.

Kelley, C. T., 1995, "Iterative Methods for Linear and Nonlinear Equations", SIAM, Philadelphia.

Pernice, M. and Walker, H. F., 1998, "NITSOL: A Newton Iterative Solver for Nonlinear Systems", SIAM J. Sci. Comput., 19(1): 302-318.

Saad, Y., 2003, "Iterative Methods for Sparse Linear Systems", SIAM Philadelphia.

Shadid, J. N., Tuminaro, R.S. and Walker, H. F., 1997, "An Inexact Newton Method for Fully Coupled Solution of the Navier-Stokes Equations with Heat and Mass Transport", J. Comp. Physics, 137: 155-185.

Tezduyar, T. E., 1991, "Stabilized Finite Element Formulations for Incompressible Flow Computations", Advances in Applied Mechanics, 28, pp. 1-44.

Tezduyar, T. E., 1999, "Finite Elements in Fluids: Lecture Notes of the Short Course on Finite Elements in Fluids", Computational Mechanics Division - vol. 99-77, Japan Society of Mechanical Engineers, Tokyo, Japan. Tezduyar, T. E., Mittal S., Ray S. E. and Shih R., 1992, "Incompressible Flow Computations with Stabilized Bilinear and Linear Equal-OrderInterpolation Velocity-Pressure Elements", Comput. Methods Appl. Mech. Engrg. 95, pp. 221-242.

Tezduyar, T. E., Aliabadi, S., Behr, M., Johnson, A. and Mittal, S., 1993, Parallel Finite Element Computation of 3D Flows, Computer, IEEE, pp. 27-36.

Tezduyar, T., Aliabadi, S., Behr, M., Johnson, A., Kalro, V. and Litke, M., 1996, "Flow Simulation and High Performance Computing", Computational Mechanics, 18, pp. 397-412.

Tuminaro, R. S., Walker, H. F. and Shadid, J. N., 2002, "On Backtracking Failure in Newton-GMRES Methods with a Demonstration for the Navier-Stokes Equations", J. Comp. Physics, 180: 549-588. 